\title{
mm-size bistable zipping dielectric elastomer actuators for integrated microfluidics
}

\author{
Luc Maffli; Samuel Rosset; Herbert R. Shea
}

Proc. SPIE 8687, Electroactive Polymer Actuators and Devices (EAPAD) 2013, 86872M (April 9, 2013); doi:10.1117/12.2009367

Copyright 2013 Society of Photo-Optical Instrumentation Engineers. One print or electronic copy may be made for personal use only. Systematic electronic or print reproduction and distribution, duplication of any material in this paper for a fee or for commercial purposes, or modification of the content of the paper are prohibited.

http://dx.doi.org/10.1117/12.2009367 


\title{
mm-size bistable zipping dielectric elastomer actuators for integrated microfluidics
}

\author{
Luc Maffli $^{1}$, Samuel Rosset and Herbert R. Shea \\ Microsystems for Space Technologies Laboratory (LMTS), Ecole Polytechnique Fédérale de \\ Lausanne (EPFL), Neuchâtel, Switzerland
}

\begin{abstract}
We report on a new structure of Dielectric Elastomer Actuators (DEAs) called zipping DEAs, which have a set of unique characteristics that are a good match for the requirements of electrically-powered integrated microfluidic pumping and/or valving units as well as Braille displays. The zipping DEAs operate by pulling electrostatically an elastomer membrane in contact with the rigid sidewalls of a sloped chamber. In this work, we report on fully functional mm-size zipping DEAs that demonstrate a complete sealing of the chamber sidewalls and a tunable bistable behavior, and compare the measurements with an analytical model. Compared to our first generation of devices, we are able vary the sidewall angle and benefit therefore from more flexibility to study the requirements to make fully functional actuators. In particular, we show that with Nusil CF19 as membrane material (1.2 MPa Young's modulus), it is possible to zip completely $2.3 \mathrm{~mm}$ diameter chambers with $15^{\circ}$ and $21^{\circ}$ sidewalls angle equibiaxially prestretched to $\lambda_{0}=1.12$ and $15^{\circ}$ chambers with $\lambda_{0}=1.27$.
\end{abstract}

Keywords : Electro Active Polymers (EAP), zipping, Dielectric Elastomer Actuators (DEA), silicone, microfluidics, Braille display, bistable

\section{INTRODUCTION}

Dielectric Elastomer Actuators (DEAs) are known since 1998 as devices exhibiting unique features, in particular very large strains ${ }^{[1,2]}$. This is possible thanks to the softness of the elastomer membrane, but this softness makes also challenging the use of DEAs to retain a precise shape when submitted to a load. For many applications, one requires outof-plane displacements in order to exert mechanical work, or to take advantage of the actuator's softness to achieve complex motions such as dielectric elastomer minimum energy structures ${ }^{[3]}$. Well beyond the simple but impressive DEA "sandwich" demonstrators, much engineering effort is required to design new actuator structures that can truly exploit all the potential of DEAs ${ }^{[4,5,6,7,8]}$.

A promising application of DEAs is to replace the pneumatic actuators of microfluidic large-scale integrated chips (MLSI) ${ }^{[9]}$ in order to enable electrically-powered portable lab-on-chips ${ }^{[10]}$ as the current MLSI systems requires large external pumps and compressed air sources. Apart from the large strains, this application imposes specific requirements on the actuator's characteristics: out-of-plane motion, sealing of embedded channels, and guaranteed complete deflection by a bistable operation mode. We proposed previously a variant of DEAs inspired by silicon MEMS zipping devices that precisely meets these requirements ${ }^{[10]}$. As a demonstration of the feasibility of DEA-powered MLSI chip, we want to develop a peristaltic micropump composed of three in-line zipping DEAs. In this work, we show a second generation of single zipping DEA devices that demonstrate experimentally the potential of this new type of actuators by achieving

\footnotetext{
${ }^{1}$ Correspondence : luc.maffli@epfl.ch
}

Electroactive Polymer Actuators and Devices (EAPAD) 2013, edited by Yoseph Bar-Cohen, Proc. of SPIE Vol. 8687, 86872M - (c) 2013 SPIE · CCC code: 0277-786X/13/\$18 · doi: 10.1117/12.2009367 
complete chamber zipping and bistable behavior. Additional characteristics of zipping DEAs include a well-controlled deflected shape and a highly tunable deflection vs. voltage characteristic including bi- or multi-stable modes, which make them first choice candidates for other applications such as Braille displays.

\section{ZIPPING DIELECTRIC ELASTOMER ACTUATORS}

As presented in our previous work ${ }^{[10,11]}$, DEA zipping actuators are a variant of the traditional DEA operation mode inspired by silicon MEMS zipping devices ${ }^{[12]}$. Instead of having two compliant electrodes that squeeze the elastomer and produce a thickness compression and lateral expansion ${ }^{[1]}$, zipping DEAs attract one single compliant electrode toward a rigid one. The elastomer membrane on which the compliant electrode is patterned deforms out-of-plane, being gradually pulled in contact with the rigid electrode (Figure 1).

Silicon MEMS zipping actuators use thin high-quality rigid dielectrics to store the electrostatic energy, which results in high forces at low voltages and made them popular ${ }^{[13]}$. As demonstrated experimentally by Gebbers et al. ${ }^{[11]}$, it is possible to fabricate DEA zipping actuators based on this same principle: the soft electrode is pulled in hard contact with the rigid dielectric, and the electric field is therefore applied across a few $\mu \mathrm{m}$ thick rigid insulator. Since solid dielectric coatings such as silicon nitride, silicon oxide or aluminum oxide have high dielectric constants and breakdown strengths, the actuation voltage can be greatly reduced. Gebbers successfully coated a $20 \mathrm{~mm}$ diameter rigid aluminium electrode with a relatively thick $(10-30 \mu \mathrm{m})$ aluminium oxide and achieved $1.4 \mathrm{~mm}$ deflection at $200 \mathrm{~V}$. Although promising, this approach is quite challenging in terms of fabrication: these very thin layers tend to have defects, so that the realization of a pinhole-free dielectric over a few square mm sloped chamber is critical. The best high-quality dielectrics are available in the well-established but expensive microfabrication technologies, however their use raises a new issue: most of their etching processes have been designed to produce vertical trenches, and are therefore badly suited to machine the sloped walls chambers.

We therefore chose to study a variant of zipping DEAs in which the electric field is applied across the elastomer membrane (Figure 1), avoiding the use of a thin rigid dielectric. We previously investigated anisotropic crystalline etching of silicon by potassium hydroxide $(\mathrm{KOH})$, which yields pyramidal-shape chambers with a large fixed sidewalls angle of $54.7^{\circ}$. We could obtain a maximal out-of plane deflection of $300 \mu \mathrm{m}$ on $2.6 \mathrm{~mm}$ side chambers covered by a membrane patterned with an $\mathrm{Au}$ ion-implanted electrode on top, not reaching the fully deflected shape $(525 \mu \mathrm{m}$ deflection) ${ }^{[10]}$. In this work, we present a new generation of devices which have conical-shape machined chambers in aluminium. Their main advantage compared with the previous design is the possibility to choose the sidewalls angle.

The devices presented here are composed of a silicone membrane with a compliant electrode patterned on top, which is suspended over a conical cavity with sloped sidewalls (Figure 1). The electric field is applied between the compliant electrode and the conducting body, which is grounded. As the voltage increases, the electrostatic force pulls the membrane in contact with the chamber sidewalls. The electric field is the highest where the gap is the smallest, and a zipping-like motion therefore proceeds from the suspended part's periphery, bringing gradually the membrane in contact with the rigid electrode. Like for silicon zipping devices, it is more stable energetically to have a fully deflected membrane above a voltage threshold: it jumps at the bottom of the cavity in a bistable fashion. 


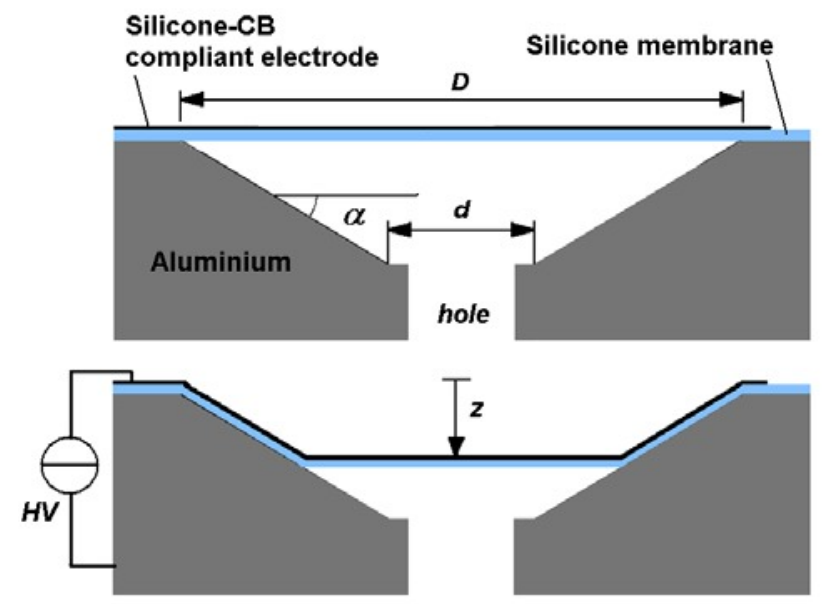

Figure 1. Cross-section of a zipping actuator consisting in a conical chamber milled in aluminium covered by a suspended membrane with a compliant electrode patterned on top. The chamber's geometrical parameters are the top and bottom diameters $D$ and $d$ and the sidewalls angle $\alpha$.

\section{ANALYTICAL PREDICTION OF THE ZIPPING BEHAVIOR}

We already proposed a model which is able to predict the static displacement of membranes zipping in pyramidal-shape chambers with $54.7^{\circ}$ sidewalls angle (corresponding to $\left.\alpha\right)^{[10]}$. We kept the same approach, changing only the chamber geometry from a pyramidal shape into a conical one. The hyperelastic Gent model parameters of the silicone elastomer (Nusil CF 19-2186) are $\mathrm{J}=80.4$ and $\mu=0.42 \mathrm{MPa}$.

\subsection{Pyramidal and conical chambers}

The Figure 2 shows the $z$ deflection versus voltage curve outputted by our model for one $\mathrm{KOH}$-etched and two conical chambers, without prestretch. Comparing the output for the $\mathrm{KOH}$-etched chamber and the conical one of same area and angle $\alpha$, one can assume that the change in geometry makes almost no difference and that it is therefore possible to compare data from both geometries to some extent. However, our model assumes all stretches to be equibiaxial, but the 3D membrane shape measurements obviously pointed out that one could expect to have inhomogeneous stretches along the edges of a pyramidal chamber ${ }^{[10]}$. We expect our new generation of axisymmetric conical-shape devices to have a stretch state that is more homogeneous. The highest machined sidewalls angle of the conical chambers is $31^{\circ}$, and we plotted it together with the two $54.7^{\circ}$ curves as a comparison. 


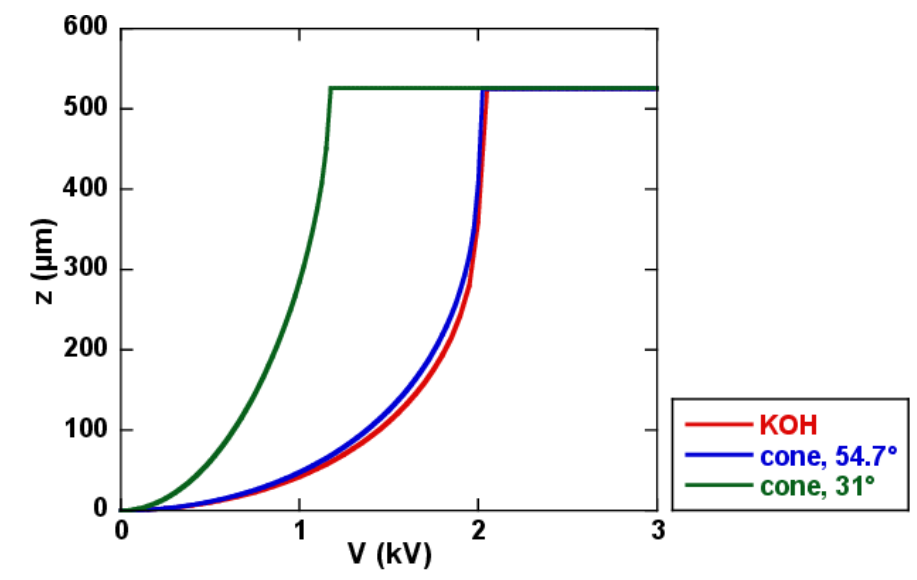

Figure 2. Model output showing zipping depth vs. applied voltage for a KOH-etched chamber and a conical chamber of same surface and angle; and a conical chamber with $31^{\circ}$ sidewalls angle. Other parameters: actuator area $4 \mathrm{~mm}^{2}$, thickness $\mathrm{t}_{0}=25 \mu \mathrm{m}, \lambda_{0}=1$ (not prestretch), maximal zipping depth set to $525 \mu \mathrm{m}$.

\subsection{Effect of membrane thickness}

The Figure 3 plots the effect of membrane thickness (after prestretch) on the zipping voltage. The breakdown points are indicated by crosses of corresponding colors. For this set of parameters, there is a regular shift of the zipping voltage of about $1.1 \mathrm{kV}$ per $20 \mu \mathrm{m}$ thickness. Increasing the membrane thickness shifts the zipping voltage by both having less electric field at a constant voltage and more mechanical energy to be compensated at a constant deflection. The breakdown voltage is slightly pushed further away from the zipping jump, and one could therefore expect a somewhat better reliability for thicker membranes.

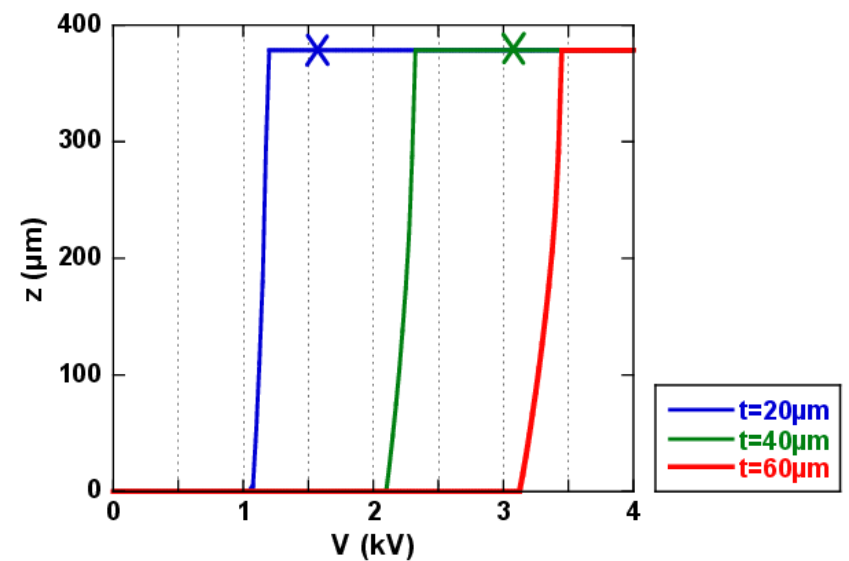

Figure 3. Simulated zipping depth vs. voltage, showing the effect of membrane thickness on $31^{\circ}$ conical chambers. The breakdown points are indicated by crosses of the corresponding colors. Other parameters: top diameter $\mathrm{D}=2.26 \mathrm{~mm}$, hole diameter $\mathrm{d}=1 \mathrm{~mm}$, prestretch $\lambda_{0}=1.12$, breakdown field of elastomer $100 \mathrm{~V} / \mu \mathrm{m}$.

\subsection{Effect of prestretch}

The Figure 4 shows the effect of increasing prestretch as predicted by the model, keeping the thickness after prestretch constant. It is twofold: it delays the zipping jump and turns its shape into a more abrupt transition. Therefore, if a very clear bistable behavior is desired, one should go to higher prestretches. It does not improve the actuation strain by 
overcoming the pull-in instability in the same fashion as traditional "squeezing-mode" DEAs, since the actuator structure is different (the zipped part of the membrane in hard contact with the sidewalls and the central part has a negligible electric field). Moreover, it must be stressed that the bistable behavior of zipping DEAs is not due to the pull-in instability but to a jump between energy minima that depends both on the actuator's geometry and on the elastomer mechanical properties.

Our first generation of chambers had been etched in a silicon wafer using anisotropic crystalline etching, which defines the large sidewalls angle of $54.7^{\circ}$. As a consequence, more voltage was needed to deflect the membrane, and we were not able to zip completely the membrane down to the $525 \mu \mathrm{m}$ depth of the wafer. However, the large sidewalls angle had the advantage that the non-prestretched membranes were able to come back in their flat position after turning off the voltage. With our current conical-shape zipping devices, we are able to fabricate chambers with lower angle $\alpha$, but it becomes mandatory to pay attention that enough mechanical restoring force is present to un-stick the membrane from the sidewalls within a fraction of second after the voltage has been switched off.

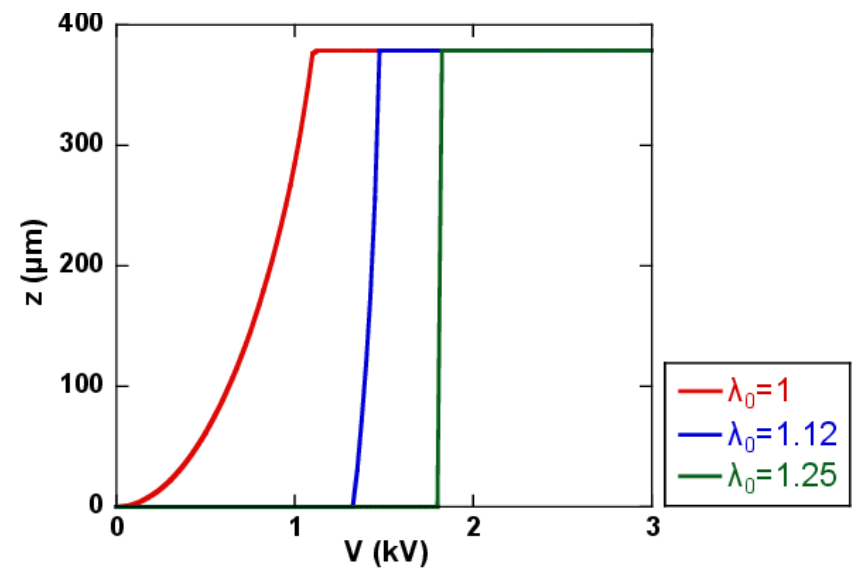

Figure 4. Simulated zipping depth vs voltage showing the effect of the prestretch $\lambda_{0}$ on $31^{\circ}$ conical chambers. Other parameters: thickness $\mathrm{t}_{0}=25 \mu \mathrm{m}$, top diameter $D=2.26 \mathrm{~mm}$, bottom diameter $d=1 \mathrm{~mm}$.

One may wonder whether it is preferable to increase the mechanical restoring force of the membrane by adding more prestretch or by using thicker membranes. From our model predictions, thicker membranes would tend to increase the security margin between the zipping jump and the breakdown. But prestretching provides a large amount of additional mechanical energy, and this without changing the thickness since we manufacture our own membranes and can therefore choose our thickness and prestretch independently. At constant voltage, using prestretch rather than thicker membrane keeps the electric field larger. To a lower extent (the involved pre-stretches are not very high for our set of parameters) it should also increase the breakdown field of the elastomer. We therefore conclude that it is preferable to increase the mechanical restoring force by prestretching rather than having thicker membranes.

\section{DEVICE FABRICATION}

The chambers were milled from an aluminium plate with a Step-Four Basic series CNC equipment. We first bored a hole in the center of the chamber, which allows the air to come out during actuation. We then use drillbits with conical shape heads of different angles to obtain the desired sidewall profiles. This machining method quickly yields chambers with axi-symmetric (conical) shape, dimensions and angles suitable for our application, but with lower surface quality and less controlled geometry than the microfabricated silicon chambers. The geometrical parameters are the sidewalls angle $\alpha$, the top diameter $D$ and the bottom diameter $d$. The sidewall angles $\alpha$ are $15^{\circ}, 22.5^{\circ}$ and $31^{\circ}$, and the top diameters comprised between 2 and $3 \mathrm{~mm}$. 
We fabricate the silicone membrane with a blade casting process which yields very homogeneous membranes of various silicone types with thicknesses from 15 to $100 \mu \mathrm{m}$. The silicone we are using in this work is CF 19-2186 from Nusil. We previously investigated the use of a softer but less breakdown resistant silicone (Bluestar LSR4305), and could zip only half of the depth obtained with the CF 19-2186 ${ }^{[14]}$. Moreover, we expect better pumping performance with stiffer silicones. Indeed, the performance of a pump is addressed in terms of backpressure and flow rate, the latter being basically defined as stroke volume multiplied by the cycle frequency. Stiffer silicones provide less stroke volume but yield higher pressures and frequencies, thus increasing the overall pumping performance.

The membranes are equibiaxially prestretched up to 1.27 to ensure that enough restoring force is stored even with small sidewalls angles to counter the stiction forces on the elastomer-aluminium interface and bring back the membrane in its flat position. Once prestretched, a compliant electrode composed of carbon black particles dispersed in a soft silicone matrix is patterned on the membrane by an indirect stamping technique (pad printing). The ink's Young's modulus is in the range of 1.9MPa, and the one of CF 19-2186 is 1.2 MPa. The use of stiffer "solid" electrodes (compared for instance to carbon black dispersed in silicon grease) requires thin layers to ensure the stiffening impact is negligible ${ }^{[15]}$. We measured electrode thicknesses between 1 to $3.5 \mu \mathrm{m}$, depending on the number of successive applications, which fits this condition.

The printed membrane is bonded over the aluminium body by activating the native oxide and the silicone surface with oxygen plasma. This method is well-known in silicone replica molding processes and produces an irreversible chemical bonding of the two parts. The activation of the surfaces decays with time, so that the suspended part of the membrane will not adhere to the sidewalls when being actuated. The compliant electrode is contacted to a PCB with a conductive varnish.

\section{EXPERIMENTAL RESULTS}

\subsection{Complete chamber zipping}

The Figure 5 shows an optical picture of a zipped $2.4 \mathrm{~mm}$ diameter actuator. The membrane completely zips onto the chamber sidewalls under a $1.5 \mathrm{kV}$ voltage.
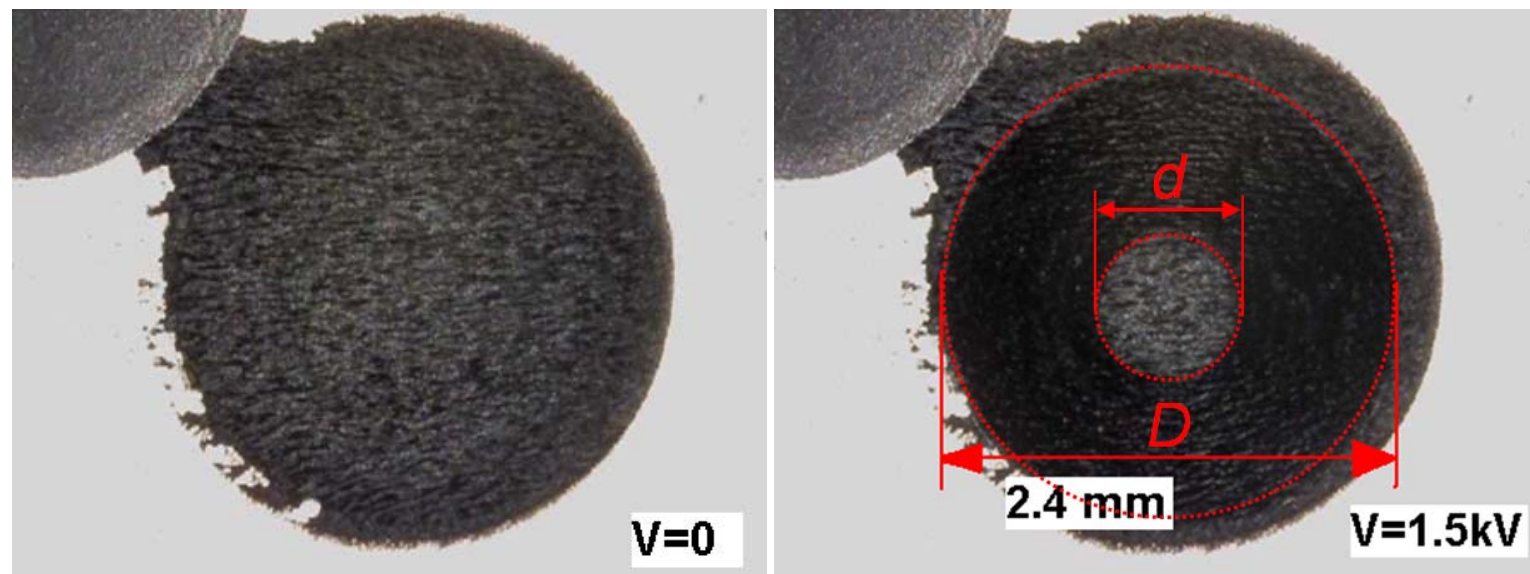

Figure 5. Optical picture of a zipping DEA, completely zipped at $1.5 \mathrm{kV}$. The. Sidewalls angle $\alpha=15^{\circ}$, equibiaxial prestretch $\lambda_{0}=1.11$, top diameter $D=2.4 \mathrm{~mm}$, membrane thickness $\mathrm{t}_{0}=23.7 \mu \mathrm{m}$. Total vertical zipping depth $z=200 \mu \mathrm{m}$. 
We show on the Figure 6 the vertical deflection $z$ versus voltage of three devices that successfully zipped down to the bottom of the chamber. They have sidewalls angles of 15 and 22.5 $5^{\circ}$, and two different prestretches. Measurement on larger angle chambers with the same prestretches could not completely zip before breakdown.

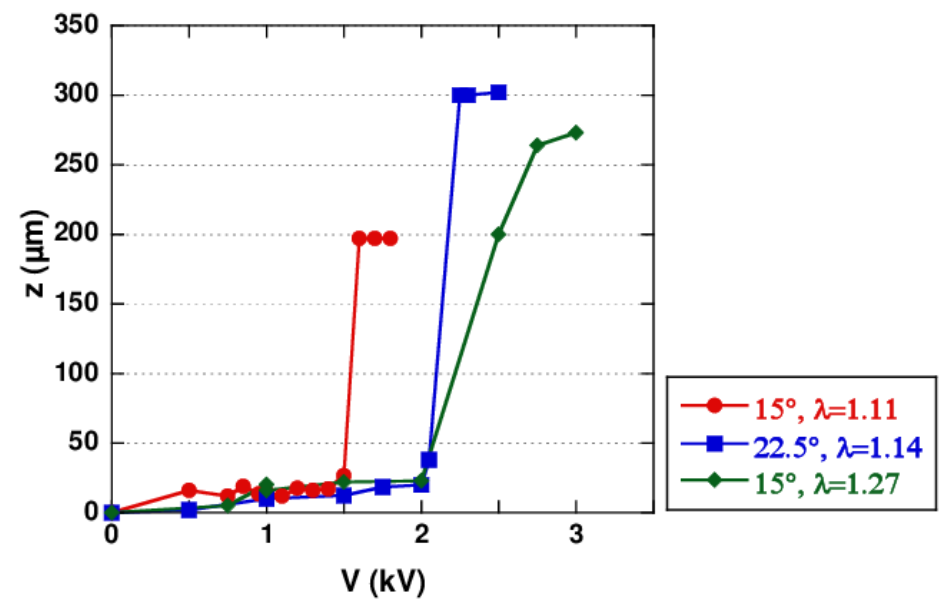

Figure 6. Experimental data showing measured zipping depth vs voltage, showing fully zipped chambers with 15 and $22.5^{\circ}$ and 2 sets of prestretches. All three chambers were able to come back to their flat position within a second. The top diameters are $D=2.31 \pm 0.15 \mathrm{~mm}$ and average thicknesses $\mathrm{t}_{0}=24.2 \pm 0.5 \mu \mathrm{m}$.

\subsection{Variation of prestretch and thickness}

Assuming that conical and pyramidal actuators of equal area should behave in the same manner (section 3.1), we compare measurement made in a $2 \mathrm{~mm}$ side $\mathrm{KOH}$-etched chamber and in a conical one (Figure 7). The $\mathrm{KOH}$-etched chamber $\left(54.7^{\circ}\right)$ has a membrane about three times thicker than the conical one, and no prestretch. The $31^{\circ}$ chamber has a small prestretch of 1.14 . Both devices failed before reaching the complete zipping depth (indicated by the flat of the model curve).

It can be seen that the measurements on the $\mathrm{KOH}$ chamber match well the model prediction, although the device had a breakdown before reaching the zipping jump. The higher maximal deflection ( $400 \mu \mathrm{m}$ vs. $300 \mu \mathrm{m}$ on previous devices) is obtained thanks to the lower stiffening impact of the CB-silicone electrode than with ion implantation ${ }^{[10]}$.

The zipping jump voltage of the conical chamber is higher than predicted by the model. A comparable difference between the model and the experiments has been observed on all the prestretched conical chambers. We attribute this difference to the fact that we did not include the bending energy of the membrane in our model. The energy needed to bend the membrane to pass from a flat to a zipped shape would add an energy barrier of at least $0.3 \mu \mathrm{J}$ amplitude (maximum mechanical stretch energy ranges from 2 to $20 \mu \mathrm{J}$ depending on the actuator's parameters) in the first 10's of $\mu \mathrm{m}$ of vertical displacement, thus delaying the zipping voltage.

The fact that the model fits better the data of the non-prestretched pyramidal shaped chamber may be attributed to the fact that the bending energy is proportional to the effective Young's modulus, and would therefore be smaller for the non-prestretched devices. 


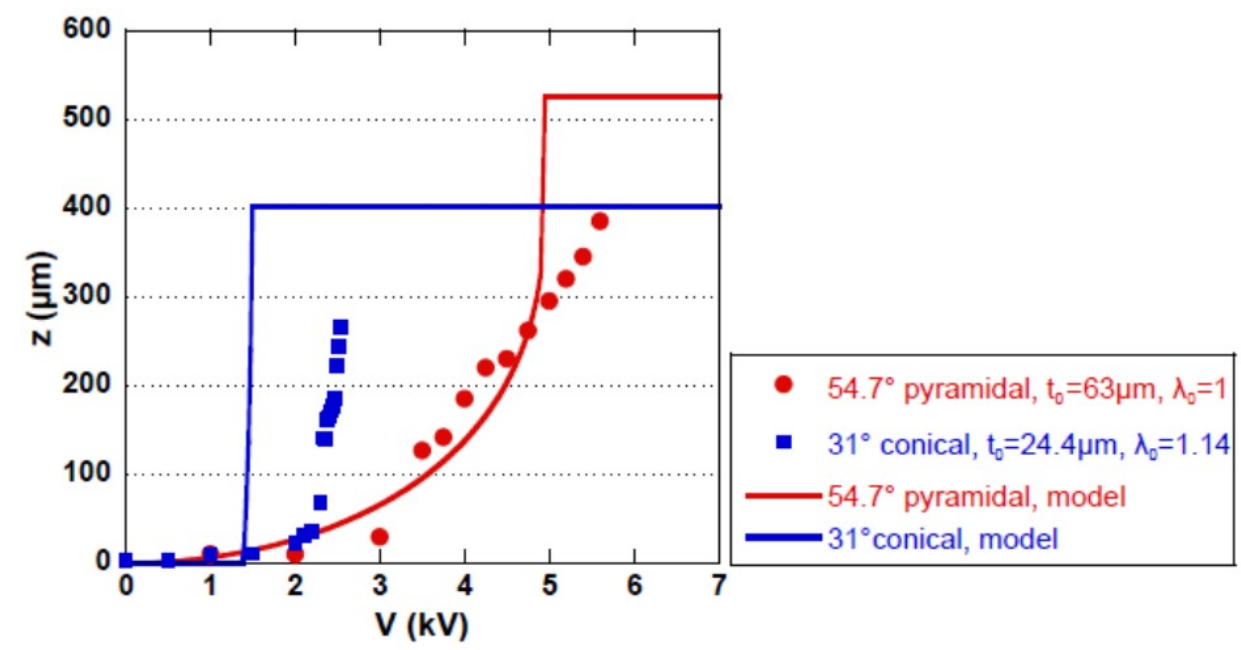

Figure 7. Model and measurement for two chambers with different thicknesses and prestretches. Pyramidal chamber: side of the top square $2 \mathrm{~mm}$. Conical chamber: to diameter $D=2.14 \mathrm{~mm}$, bottom diameter $d=0.8 \mathrm{~mm}$.

The Figure 8 displays the zipping data of two $22.5^{\circ}$ sidewalls conical-shape actuators and their corresponding model prediction. The first device (red) has a thickness of $31 \mu \mathrm{m}$ and a prestretch of 1.12, and the second (blue) $25 \mu \mathrm{m}$ thickness and a prestretch of 1.27 . The small difference of $0.15 \mathrm{~mm}$ in top diameter does not change the zipping voltage visibly (but the maximal zipping depth). As explained earlier, the model underestimates the zipping voltages because of the neglected bending energy. The thickness and prestretch are two counter-acting effects regarding the zipping voltage. According to the model, the effect of the thickness difference dominates over the prestretch, and the zipping occurs $0.25 \mathrm{kV}$ earlier with the thinner actuator. This prediction is confirmed by the measurements, which have exactly this same difference in zipping jump voltage.

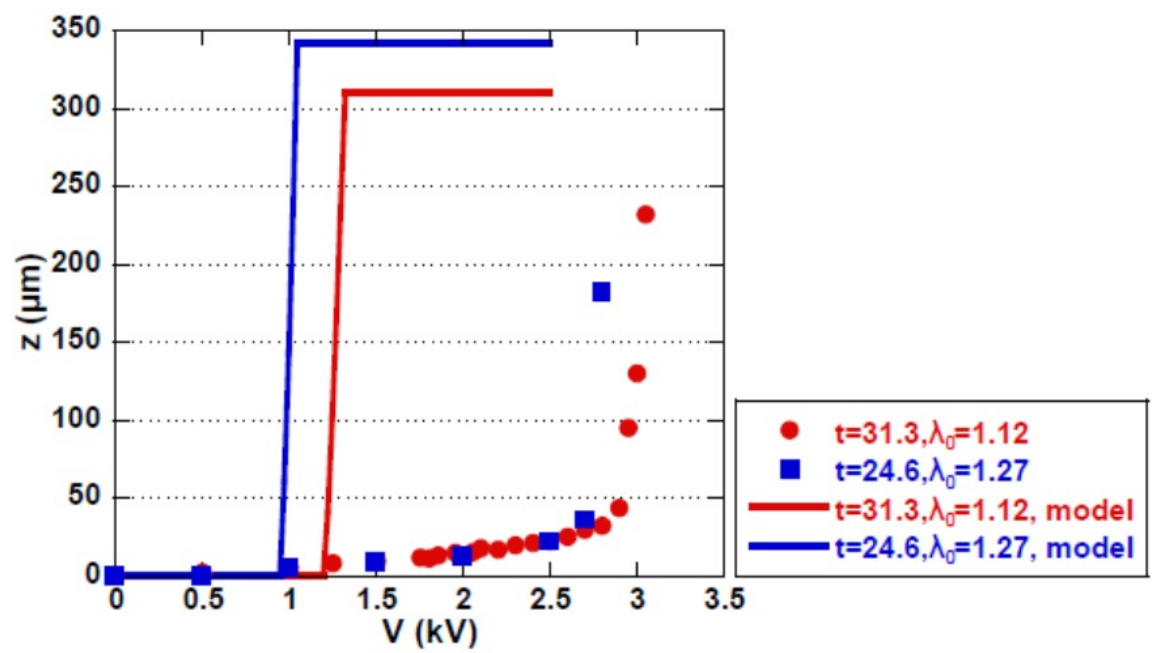

Figure 8. Model and measurement for two $22.5^{\circ}$ conical chambers with different thicknesses and prestretches. The small difference in top diameters $D(2.3$ and $2.45 \mathrm{~mm})$ plays a negligible role regarding the zipping voltage but on the maximal zipping depth. The model predicts well the shift of the zipping voltage of about $0.25 \mathrm{kV}$, which is dominated by the different membrane thickness rather than the prestretch. Bottom diameter $d=0.8 \mathrm{~mm}$. 


\section{CONCLUSIONS}

Zipping DEAs are an emerging class of dielectric elastomer actuators which deform a soft elastomer membrane covered with a compliant electrode by pulling it electrostatically inside a chamber with sloped sidewalls. They exhibit unique features such as well-controlled out-of-plane deflected shape, sidewalls sealing and highly tunable bistable behavior. Unlike our first generation of devices, the actuators presented here are composed of conical chambers milled in an aluminium plate, which allows a study in which we vary the sidewall angle. We present experimental results showing fully functional bistable zipping actuators and compare these data with a previously developed analytical model. $25 \mu \mathrm{m}$ thick silicone membranes equibiaxially prestretched to $\lambda_{0}=1.12$ with 1.2 MPa Young's modulus (CF 19-2186 from Nusil) zip completely down in $2.3 \mathrm{~mm}$ diameter chambers with $15^{\circ}$ and $21^{\circ}$ sidewalls angle. With a larger prestretch $\left(\lambda_{0}=1.27\right)$, only the $15^{\circ}$ chambers were able to zip completely without breakdown. The comparison of the two datasets with a model developed in a previous work indicates that the combined effect of membrane thickness and prestretch variation on the zipping jump voltage is well predicted, although a systematic shift of the zipping jump voltage between measurement and data is observed. This shift is mostly due to having not included the bending energy in the model. This new generation of actuators would fit the requirements to replace the large-displacement pneumatic valves of large-scale integrated chips or Braille displays.

\section{ACKNOWLEDGMENTS}

The authors would like to thank Mrs Samin Akbari, Dr. Seun Araromi, Mr. Jun Shintake and Mr. Alexandre Poulin for their precious collaboration. Financial support is gratefully acknowledged from the Swiss National Science Foundation, grant \# 200020-140394.

\section{REFERENCES}

[1] R. E. Pelrine, R. D. Kornbluh and J. P. Joseph, "Electrostriction of polymer dielectrics with compliant electrodes as a means of actuation," Sensors and Actuators A: Physical, vol. 64, no. 1, pp. 77-85 (1998)

[2] C. Keplinger, T. Li, R. Baumgartner, Z. Suo and S. Bauer, "Harnessing snap-through instability in soft dielectrics to achieve giant voltage-triggered deformation," Soft Matter, vol. 8, no. 2, pp. 285-288 (2012)

[3] G. Kofod, W. Wirges, M. Paajanen and S. Bauer, "Energy minimization for self-organized structure formation and actuation," Applied Physics Letters, vol. 90 (2007)

[4] S. Akbari and H. Shea, "An array of $100 \mu \mathrm{m} \times 100 \mu \mathrm{m}$ dielectric elastomer actuators with $80 \%$ strain for tissue engineering applications," Sensors and Actuators A: Physical, vol. 186, pp. 236-241 (2012)

[5] I. A. Anderson, T. A. Gisby, T. G. McKay, B. M. O'Brien and E. P. Calius, "Multi-functional dielectric elastomer artificial muscles for soft and smart machines," Journal of Applied Physics, vol. 112, no. 4, pp. 041101 - 041101-20 (2012)

[6] F. Carpi, C. Salaris and D. D. Rossi, "Folded Dielectric Elastomer Actuators," Smart Mater. Struct., vol. 16, pp. S300-S305 (2007) 
[7] R. Kornbluh, "Fundamental Configurations for Dielectric Elastomer Actuators," in Dielectric Elastomers as Electromechanical Transducers, Oxford, Elsevier, pp. 79-90 (2008)

[8] S. Rosset, M. Niklaus, P. Dubois and H. R. Shea, "Large-Stroke Dielectric Elastomer Actuators With Ion-Implanted Electrodes," Journal of Microelectromechanical Systems, vol. 18, no. 6, pp. 1300-1308 (2009)

[9] T. Thorsen, S. J. Maerkl and S. R. Quake, "Microfluidic Large-Scale Integration," Science, vol. 298, pp. 580-584 (2002)

[10] L. Maffli, B. O'Brien, S. Rosset and H. Shea, "Pump it up," in Proc. of SPIE, Vol. 8340, 83402Q (2012)

[11] P. Gebbers, C. Grätzel, L. Maffli, C. Stamm and H. Shea, "Zipping it up: DEAs independent of the elastomer's electric breakdown field," in Proc. of SPIE, Vol. 8340, 83402P-1 (2012)

[12] J. Branebjerg and P. Gravesen, "A New Electrostatic Actuator providing improved Stroke length and Force," in Micro Electro Mechanical Systems, Travelmünde (Germany) (1992)

[13] J. Li, Electrostatic Zipping Actuators and Their Application to MEMS, Massachusetts Institute of Technology (2004)

[14] L. Maffli, S. Rosset, H. R. Shea, "Zipping Dielectric Elastomer Actuators for microfluidics," in EuroEAP International conference on EAP transducers \& artificial muscles, Potsdam (2012)

[15] S. Rosset and H. Shea, "Flexible and stretchable electrodes for dielectric elastomer actuators," Applied Physics A, vol. 110, no. 2, pp. 281-307 (2013) 